\title{
On invariants of curves in centro-affine geometry
}

\author{
By \\ Ömer PeKşen and Djavvat KhadJIEv
}

\begin{abstract}
Let $G L(n, R)$ be the general linear group of $n \times n$ real matrices. Definitions of $G L(n, R)$-equivalence and the centro-affine type of curves are introduced. All possible centro-affine types are founded. For every centro affine type all invariant parametrizations of a curve are described. The problem of $G L(n, R)$-equivalence of curves is reduced to that of paths. A generating system of the differential field of invariant differential rational functions of a path is described. They can be viewed as centro-affine curvatures of a path. Global conditions of $G L(n, R)$ equivalence of curves are given in terms of the centro-affine type and the generating differential invariants. Independence of elements of the generating differential invariants is proved.
\end{abstract}

\section{Introduction}

The fundamental theorem of curves in $n$-dimensional centro-affine geometry is obtained by Gardner and Wilkens [5]. In the paper they used Cartan's method [4] of moving frames in order to find the formulation of the local rigidity theorem for curves that is amenable to direct application to problems in control theory. They provide a method for constructing the centro-affine curvatures $\kappa_{1}(s), \ldots, \kappa_{n}(s)$. They have obtained explicit formulae for computing a centroaffine arclength in terms of an arbitrary parameter and the first curvature $\kappa_{1}(s)$. In this paper there are no explicit formulae for centro-affine curvatures $\kappa_{2}(s), \ldots, \kappa_{n}(s)$, but their orders are defined. A discussion of centro-affine plane curves, as well as a very brief discussion of centro-affine space curves, can be found in ([15], [13], [17]). A very detailed discussion of the centro-affine theory of plane curves can be found in Laugwitz [11]. The first comprehensive treatment of affine geometry is given in the seminal work of Blaschke [3]. For further developments of the subject, we refer the reader to [14], and the more modern texts [20], [12], commentaries [16], [17], and survey papers [19], [2],

1991 Mathematics Subject Classification(s). 53A15, 53A55

This work was supported by the Research Fund of Karadeniz Technical University. Project number: 20.111.003.4

Received February 25, 2004 
[18]. Equi-affine invariants of 3-dimensional space curves are investigated by Izumiya and Sano [7]. For curvatures of curves in $n$-dimensional equi-affine geometry see ([6, pp. 170-172], [13]). But in all these works, equivalence of curves is investigated locally. The global $S L(n)$-equivalence of paths in $R^{n}$ and $C^{n}$ is considered by Khadjiev [8] and Suhtaeva [21]. Complete systems of global equi-affine invariants for plane and space paths are obtained by Angelis, Moons, Van Gool and Verstraelen [1]. The complete system of global differential and integral invariants for curves in $n$-dimensional equi-affine geometry is obtained by Khadjiev and Pekşen [9].

Our paper is concerned with the problem of the global equivalence of centro-affine curves. We introduce a centro-affine type of a curve. The centroaffine type of a curve coincides with the centro-affine arclength if it is finite. But curves with infinite centro-affine arclength have three different centro-affine types. We describe all possible invariant parametrizations of a curve for every centro-affine type. We obtained a generating system of the differential field of all centro-affine invariant differential rational functions of a path. We can consider elements of the generating system as curvatures of a path. Our curvatures coincide with $c_{1}(s), \ldots, c_{n}(s)$ functions of Gardner and Wilkens ([5, p. 398]). We give the explicit formulae of curvatures in terms of the centro-affine invariant parameter, the conditions of the global centro-affine equivalence of curves in terms of the centro-affine type and curvatures of a curve. We prove an independence of curvatures.

\section{The centro-affine type of a curve and the theorem on reduction}

Let $R$ be the field of real numbers and $I=(a, b)$ be an open interval of $R$.

Definition 2.1. $\quad$ A $C^{\infty}$-map $x: I \rightarrow R^{n}$ will be called an $I$-path (shortly, a path) in $R^{n}$.

Definition 2.2. An $I_{1}$-path $x(t)$ and an $I_{2}$-path $y(r)$ in $R^{n}$ will be called $D$-equivalent if there exists a $C^{\infty}$-diffeomorphism $\varphi: I_{2} \rightarrow I_{1}$ such that $\varphi^{\prime}(r)>0$ and $y(r)=x(\varphi(r))$ for all $r \in I_{2}$. A class of $D$-equivalent paths in $R^{n}$ will be called a curve in $R^{n}$, ([10, p. 9]). A path $x \in \alpha$ will be called a parametrization of a curve $\alpha$.

Remark 1. There exist different definitions of a curve ([6, p. 2], [8]).

Let $G=G L(n, R)$ be the general linear group of $n \times n$ regular matrices. $G$ acts by $(g, x) \rightarrow g x$ on $R^{n}$, where $g x$ is the multiplication of a matrix $g$ and a column vector $x \in R^{n}$.

If $x(t)$ is an $I$-path in $R^{n}$ then $g x(t)$ is an $I$-path in $R^{n}$ for any $g \in G$.

Definition 2.3. Two $I$-paths $x$ and $y$ in $R^{n}$ will be called $G$-equivalent and written $x \stackrel{G}{\sim} y$ if there exists $g \in G$ such that $y(t)=g x(t)$.

Let $\alpha$ be a curve in $R^{n}$, that is, $\alpha=\left\{h_{\tau}, \tau \in Q\right\}$, where $h_{\tau}$ is a parametrization of $\alpha$. Then $g \alpha=\left\{g h_{\tau}, \tau \in Q\right\}$ is a curve in $R^{n}$ for any $g \in G$. 
Definition 2.4. $\quad$ Two curves $\alpha$ and $\beta$ in $R^{n}$ will be called $G$-equivalent (or $G$-congruent) and written $\alpha \stackrel{G}{\sim} \beta$ if $\beta=g \alpha$ for some $g \in G$.

Remark 2. Our definition is essentially different from the definition ([6, p. 21]) of a congruence of curves for the group of euclidean motions. By the definition ([6, p. 21]), two curves with different lengths may be congruent.

Let $x$ be an $I$-path in $R^{n}$ and $x^{\prime}(t)$ be the derivative of $x(t)$. Put $x^{(0)}=$ $x, x^{(n)}=\left(x^{(n-1)}\right)^{\prime}$. For $a_{k} \in R^{n}, k=1, \ldots, n$, the $\operatorname{determinant} \operatorname{det}\left(a_{i j}\right)$ (where $a_{k i}$ are coordinates of $a_{k}$ ) will be denoted by $\left[a_{1} a_{2} \ldots a_{n}\right]$. So $\left[x(t) x^{\prime}(t) \ldots x^{(n-1)}(t)\right]$ is the determinant of the vectors $x(t), x^{\prime}(t), \ldots$, $x^{(n-1)}(t)$.

Definition 2.5. An $I$-path $x(t)$ in $R^{n}$ will be called substantial if both

$$
\left[x(t) x^{\prime}(t) \ldots x^{(n-1)}(t)\right] \neq 0 \text { and }\left[x^{(n)}(t) x^{\prime}(t) \ldots x^{(n-1)}(t)\right] \neq 0
$$

for all $t \in I$. A curve will be called substantial if it contains a substantial path [5].

For $I=(a, b), q, p \in I$ and a substantional $I$-path $x(t)$ put

$$
l_{x}(q, p)=\int_{q}^{p}\left|\frac{\left[x^{(n)}(t) x^{\prime}(t) \ldots x^{(n-1)}(t)\right]}{\left[x(t) x^{\prime}(t) \ldots x^{(n-1)}(t)\right]}\right|^{\frac{1}{n}} d t
$$

and $l_{x}(a, p)=\lim _{q \rightarrow a} l_{x}(q, p), l_{x}(q, b)=\lim _{p \rightarrow b} l_{x}(q, p)$. There are only four possible cases:

(i) $l_{x}(a, p)<+\infty, l_{x}(q, b)<+\infty$; $(i i) l_{x}(a, p)<+\infty, l_{x}(q, b)=+\infty$;

(iii) $l_{x}(a, p)=+\infty, l_{x}(q, b)<+\infty$; $($ iv $) l_{x}(a, p)=+\infty, l_{x}(q, b)=+\infty$.

Suppose that the case $(i)$ or $(i i)$ holds for some $q, p \in I$. Then the number $l=l_{x}(a, p)+l_{x}(q, b)-l_{x}(q, p)$, where $0 \leq l \leq+\infty$, does not depend on $q, p$. In this case, we say that $x$ belongs to the centro-affine type of $(0, l)$. The cases (iii) and (iv) do not depend on $q, p$. In these cases, we say that $x$ belongs to the centro-affine types of $(-\infty, 0)$ and $(-\infty,+\infty)$, respectively. There exist paths of all types $(0, l)$ (where $0 \leq l \leq+\infty),(-\infty, 0)$ and $(-\infty,+\infty)$. The centro-affine type of a path $x$ will be denoted by $L(x)$.

\section{Proposition 2.1.}

(i) If $x \stackrel{G}{\sim} y$ then $L(x)=L(y)$;

(ii) Let $\alpha$ be a curve and $x, y \in \alpha$. Then $L(x)=L(y)$.

Proof. It is obvious.

The centro-affine type of a path $x \in \alpha$ will be called the centro-affine type of the curve $\alpha$ and denoted by $L(\alpha)$. According to Proposition 2.1, $L(\alpha)$ is a $G$-invariant of a curve $\alpha$. 
Now we define an invariant parametrization of a substantial curve in $R^{n}$.

Let $I=(a, b)$ and $x(t)$ be a substantial $I$-path in $R^{n}$. We define the centroaffine arc length function $s_{x}(t)$ for each centro-affine type as follows. We put $s_{x}(t)=l_{x}(a, t)$ for the case $L(x)=(0, l)$, where $0<l \leq+\infty$, and $s_{x}(t)=-$ $l_{x}(t, b)$ for the case $L(x)=(-\infty, 0)$. Let $L(x)=(-\infty,+\infty)$. We choose a fixed point in every interval $I=(a, b)$ of $R$ and denote it by $a_{I}$. Let $a_{I}=0$ for $I=(-\infty,+\infty)$. We set $s_{x}(t)=l_{x}\left(a_{I}, t\right)$.

Since $s_{x}^{\prime}(t)>0$ for all $t \in I$, the inverse function of $s_{x}(t)$ exists. Let us denote it by $t_{x}(s)$. The domain of $t_{x}(s)$ is $L(x)$ and $t_{x}^{\prime}(s)>0$ for all $s \in L(x)$. Then

Proposition 2.2. $\quad$ Let $I=(a, b)$ and $x$ be a substantial $I$-path in $R^{n}$.

(i) $s_{g x}(t)=s_{x}(t)$ and $t_{g x}(s)=t_{x}(s)$ for all $g \in G$;

(ii) the equalities $s_{x(\varphi)}(r)=s_{x}(\varphi(r))+s_{0}$ and $\varphi\left(t_{x(\varphi)}\left(s+s_{0}\right)\right)=t_{x}(s)$ hold for any $C^{\infty}$-diffeomorphism $\varphi: J=(c, d) \rightarrow I$ such that $\varphi^{\prime}(r)>0$ for all $r \in J$, where $s_{0}=0$ for $L(x) \neq(-\infty,+\infty)$ and $s_{0}=l_{x}\left(\varphi\left(a_{J}\right), a_{I}\right)$ for $L(x)=(-\infty,+\infty)$.

Proof. $\quad(i)$. Let $L(x)=(0, l)$, where $0<l \leq+\infty$. Then we have

$$
\begin{aligned}
s_{g x}(t) & =\lim _{t_{0} \rightarrow a^{+}} \int_{t_{0}}^{t}\left|\frac{\left[(g x)^{(n)}(t)(g x)^{\prime}(t) \ldots(g x)^{(n-1)}(t)\right]}{\left[(g x)(t)(g x)^{\prime}(t) \ldots(g x)^{(n-1)}(t)\right]}\right|^{\frac{1}{n}} d t \\
& =\lim _{t_{0} \rightarrow a^{+}} \int_{t_{0}}^{t}\left|\frac{\left[x^{(n)}(t) x^{\prime}(t) \ldots x^{(n-1)}(t)\right]}{\left[x(t) x^{\prime}(t) \ldots x^{(n-1)}(t)\right]}\right|^{\frac{1}{n}} d t \\
& =s_{x}(t) .
\end{aligned}
$$

For the second part, we obtain $s_{g x}\left(t_{g x}(s)\right)=s, t_{g x}\left(s_{g x}(t)\right)=t, s_{g x}\left(t_{g x}(s)\right)=$ $s_{x}\left(t_{g x}(s)\right)=s, t_{g x}\left(s_{g x}(t)\right)=t_{g x}\left(s_{x}(t)\right)=t$. Therefore $t_{g x}(s)=t_{x}(s)$. Proofs of $(i)$ for centro-affine types $(-\infty, 0)$ and $(-\infty,+\infty)$ are similar.

For $($ ii $)$ let $L(x)=(-\infty,+\infty)$. Then we have

$$
\begin{aligned}
s_{x(\varphi)}(r) & =\int_{a_{J}}^{r}\left|\frac{\left[\frac{d^{n}}{d r^{n}} x(\varphi(r)) \frac{d}{d r}(x(\varphi(r))) \ldots \frac{d^{n-1}}{d r^{n-1}}(x(\varphi(r)))\right]}{\left[x(\varphi(r)) \frac{d}{d r}(x(\varphi(r))) \ldots \frac{d^{n-1}}{d r^{n-1}}(x(\varphi(r)))\right]}\right|^{\frac{1}{n}} d r \\
& =\int_{a_{J}}^{r} \frac{d \varphi}{d r}\left|\frac{\left[\frac{d^{n}}{d \varphi^{n}} x(\varphi(r)) \frac{d}{d \varphi}(x(\varphi(r))) \ldots \frac{d^{n-1}}{d \varphi^{n-1}}(x(\varphi(r)))\right]}{\left[x(\varphi(r)) \frac{d}{d \varphi}(x(\varphi(r))) \ldots \frac{d^{n-1}}{d \varphi^{n-1}}(x(\varphi(r)))\right]}\right|^{\frac{1}{n}} d r \\
& =l_{x}\left(\varphi\left(a_{J}\right), \varphi(r)\right)=l_{x}\left(a_{I}, \varphi(r)\right)+l_{x}\left(\varphi\left(a_{J}\right), a_{I}\right) .
\end{aligned}
$$

So $s_{x(\varphi)}(r)=s_{x}(\varphi(r))+s_{0}$, where $s_{0}=l_{x}\left(\varphi\left(a_{J}\right), a_{I}\right)$. This implies that $\varphi\left(t_{x(\varphi)}\left(s+s_{0}\right)\right)=t_{x}(s)$. For $L(x) \neq(-\infty,+\infty)$, it is easy to see that $s_{0}=0$. 
Let $\alpha$ be a substantial curve and $x \in \alpha$. Then $x\left(t_{x}(s)\right)$ is a parametrization of $\alpha$.

Definition 2.6. The parametrization $x\left(t_{x}(s)\right)$ of a substantial curve $\alpha$ will be called an invariant parametrization of $\alpha$.

We denote the set of all invariant parametrizations of $\alpha$ by $\phi_{\alpha}$. Every $y \in \phi_{\alpha}$ is an $I$-path, where $I=L(\alpha)$.

Proposition 2.3. Let $\alpha$ be a substantial curve, $x \in \alpha$ and $x$ be an $I$-path, where $I=L(\alpha)$. Then the following conditions are equivalent:

(i) $x$ is an invariant parametrization of $\alpha$;

(ii) $\left|\frac{\left[x^{(n)}(s) x^{\prime}(t) \ldots x^{(n-1)}(s)\right]}{\left[x(s) x^{\prime}(s) \ldots x^{(n-1)}(s)\right]}\right|=1$ for all $s \in L(\alpha)$;

(iii) $s_{x}(s)=s$ for all $s \in L(\alpha)$.

Proof. $\quad(i) \Rightarrow($ ii $)$. Let $x \in \phi_{\alpha}$. Then there exists $y \in \alpha$ such that $x(s)=y\left(t_{y}(s)\right)$. By Proposition 2.2, $s_{x}(s)=s_{y\left(t_{y}\right)}(s)=s_{y}\left(t_{y}(s)\right)+s_{0}=$ $s+s_{0}$, where $s_{0}$ is as in Proposition 2.2. Since $s_{0}$ does not depend on $s$, $\frac{d s_{x}(s)}{d s}=\left|\frac{\left[x^{(n)}(s) x^{\prime}(t) \ldots x^{(n-1)}(s)\right]}{\left[x(s) x^{\prime}(s) \ldots x^{(n-1)}(s)\right]}\right|^{\frac{1}{n}}=1$. Hence $\left|\frac{\left[x^{(n)}(s) x^{\prime}(t) \ldots x^{(n-1)}(s)\right]}{\left[x(s) x^{\prime}(s) \ldots x^{(n-1)}(s)\right]}\right|=1$ for all $s \in L(\alpha)$.

$(i i) \Rightarrow($ iii $)$. Let $\left|\frac{\left[x^{(n)}(s) x^{\prime}(t) \ldots x^{(n-1)}(s)\right]}{\left[x(s) x^{\prime}(s) \ldots x^{(n-1)}(s)\right]}\right|=1$ for all $s \in L(\alpha)$. By the definition of $s_{x}(t)$, we have $\frac{d s_{x}(s)}{d s}=\left|\frac{\left[x^{(n)}(s) x^{\prime}(t) \ldots x^{(n-1)}(s)\right]}{\left[x(s) x^{\prime}(s) \ldots x^{(n-1)}(s)\right]}\right|^{\frac{1}{n}}=1$. Therefore $s_{x}(s)=s+c$ for some $c \in R$. In the case $L(x) \neq(-\infty,+\infty), s_{x}(s)=s+c$ and $s_{x}(s) \in L(\alpha)$ for all $s \in L(\alpha)$ implies $c=0$, that is, $s_{x}(s)=s$. In the case $L(x)=(-\infty,+\infty), s_{x}(s)=l_{x}\left(a_{I}, s\right)=l_{x}(0, s)=s+c$ implies $0=l_{x}(0,0)=c$, that is, $s_{x}(s)=s$.

$($ iii $) \Rightarrow(i)$. The equality $s_{x}(s)=s$ implies $t_{x}(s)=s$. Therefore $x(s)=$ $x\left(t_{x}(s)\right) \in \phi_{\alpha}$.

Proposition 2.4. Let $\alpha$ be a substantial curve and $L(\alpha) \neq(-\infty,+\infty)$. Then there exists the unique invariant parametrization of $\alpha$.

Proof. Let $x, y \in \alpha, x$ be an $I_{1}$-path and $y$ be an $I_{2}$-path. Then there exists a $C^{\infty}$-diffeomorphism $\varphi: I_{2} \rightarrow I_{1}$ such that $\varphi^{\prime}(r)>0$ and $y(r)=x(\varphi(r))$ for all $r \in I_{2}$. By Proposition 2.2 and $L(\alpha) \neq(-\infty,+\infty)$, we obtain $y\left(t_{y}(s)\right)=$ $x\left(\varphi\left(t_{y}(s)\right)=x\left(\varphi\left(t_{x(\varphi)}(s)\right)\right)=x\left(t_{x}(s)\right)\right.$.

Let $\alpha$ be a substantial curve and $L(\alpha)=(-\infty,+\infty)$. Then it is easy to see that the set $\phi_{\alpha}$ is not countable.

Proposition 2.5. Let $\alpha$ be a substantial curve, $L(\alpha)=(-\infty,+\infty)$ and $x \in \phi_{\alpha}$. Then $\phi_{\alpha}=\left\{y: y(s)=x\left(s+s^{\prime}\right), s^{\prime} \in(-\infty,+\infty)\right\}$. 
Proof. Let $x, y \in \phi_{\alpha}$. Then there exist $h, k \in \alpha$ such that $x(s)=h\left(t_{h}(s)\right)$, $y(s)=k\left(t_{k}(s)\right)$, where $h$ is an $I_{1}$-path and $k$ is an $I_{2}$-path. Since $h, k \in \alpha$ there exists $\varphi: I_{2} \rightarrow I_{1}$ such that $\varphi^{\prime}(r)>0$ and $k(r)=h(\varphi(r))$ for all $r \in I_{2}$. By Proposition 2.2, $y(s)=k\left(t_{k}(s)\right)=h\left(\varphi\left(t_{k}(s)\right)=h\left(\varphi\left(t_{h(\varphi)}(s)\right)\right)=\right.$ $h\left(t_{h}\left(s-s_{0}\right)\right)=x\left(s-s_{0}\right)$.

Let $x \in \phi_{\alpha}$ and $s^{\prime} \in(-\infty,+\infty)$. We prove $x(\psi) \in \phi_{\alpha}$, where $\psi(s)=s+s^{\prime}$. By Proposition 2.3, $\left|\frac{\left[x^{(n)}(s) x^{\prime}(t) \ldots x^{(n-1)}(s)\right]}{\left[x(s) x^{\prime}(s) \ldots x^{(n-1)}(s)\right]}\right|=1$ and $s_{x}(s)=s$. Put $z(s)=$ $x(\psi(s))$. Since $\psi$ is a $C^{\infty}$-diffeomorphism of $(-\infty,+\infty)$ onto $(-\infty,+\infty)$, then $z=x(\psi) \in \alpha$. Using Proposition 2.2 and $s_{x}(s)=s$, we get $s_{z}(s)=s_{x(\psi)}(s)=$ $s_{x}(\psi(s))+s_{1}=\left(s+s^{\prime}\right)+s_{1}$, where

$$
s_{1}=\int_{\psi(0)}^{0}\left|\frac{\left[x^{(n)}(s) x^{\prime}(t) \ldots x^{(n-1)}(s)\right]}{\left[x(s) x^{\prime}(s) \ldots x^{(n-1)}(s)\right]}\right|^{\frac{1}{n}} d s .
$$

This, in view of $\left|\frac{\left[x^{(n)}(s) x^{\prime}(t) \ldots x^{(n-1)}(s)\right]}{\left[x(s) x^{\prime}(s) \ldots x^{(n-1)}(s)\right]}\right|=1$, implies $s_{1}=-\psi(0)=-s^{\prime}$. Then $s_{z}(s)=\left(s+s^{\prime}\right)-s^{\prime}=s$. By Proposition 2.3, $z \in \phi_{\alpha}$.

Theorem 2.1. $\quad$ Let $\alpha, \beta$ be substantial curves and $x \in \phi_{\alpha}, y \in \phi_{\beta}$. Then,

(i) for $L(\alpha)=L(\beta) \neq(-\infty,+\infty), \alpha \stackrel{G}{\sim} \beta$ if and only if $x(s) \stackrel{G}{\sim} y(s)$;

(ii) for $L(\alpha)=L(\beta)=(-\infty,+\infty), \alpha \stackrel{G}{\sim} \beta$ if and only if $x(s) \stackrel{G}{\sim} y\left(s+s^{\prime}\right)$ for some $s^{\prime} \in(-\infty,+\infty)$.

Proof. (i) Let $\alpha \stackrel{G}{\sim} \beta$ and $h \in \alpha$. Then there exists $g \in G$ such that $\beta=g \alpha$. This implies $g h \in \beta$. Using Propositions 2.2 and 2.4, we get $x(s)=h\left(t_{h}(s)\right), y(s)=(g h)\left(t_{g h}(s)\right)$ and $g x(s)=g\left(h\left(t_{h}(s)\right)\right)=(g h)\left(t_{h}(s)\right)=$ $(g h)\left(t_{g h}(s)\right)=y(s)$. Thus $x \stackrel{G}{\sim} y$. Conversely, let $x \stackrel{G}{\sim} y$, that is, there exists $g \in G$ such that $g x=y$. Then $\alpha \stackrel{G}{\sim} \beta$.

(ii) Let $\alpha \stackrel{G}{\sim} \beta$. Then there exist $I$-paths $h \in \alpha, k \in \beta$ and $g \in G$ such that $k(t)=g h(t)$. We have $k\left(t_{k}(s)\right)=k\left(t_{g h}(s)\right)=k\left(t_{h}(s)\right)=(g h)\left(t_{h}(s)\right)$. By Proposition 2.5, $x(s)=k\left(t_{k}\left(s+s_{1}\right)\right), y(s)=h\left(t_{h}\left(s+s_{2}\right)\right)$ for some $s_{1}$, $s_{2} \in(-\infty,+\infty)$. Therefore $x\left(s-s_{1}\right)=g y\left(s-s_{2}\right)$. This implies that $x(s) \stackrel{G}{\sim}$ $y\left(s+s^{\prime}\right)$, where $s^{\prime}=s_{1}-s_{2}$. Conversely, let $x(s) \stackrel{G}{\sim} y\left(s+s^{\prime}\right)$ for some $s^{\prime} \in$ $(-\infty,+\infty)$. Then there exists $g \in G$ such that $y\left(s+s^{\prime}\right)=g x(s)$. Since $y\left(s+s^{\prime}\right) \in \beta$, then $\alpha \stackrel{G}{\sim} \beta$.

Definition 2.7. Two $I$-paths $x$ and $y$ in $R^{n}$, where $I=R$, will be called $(G, R)$-equivalent if there exists $g \in G$ and $s^{\prime} \in R=(-\infty,+\infty)$ such that $y(s)=g x\left(s+s^{\prime}\right)$ for all $s \in(-\infty,+\infty)$.

Theorem 2.1 reduces the problem of the $G$-equivalence of substantial curves to that of paths for the case $L(\alpha)=L(\beta) \neq(-\infty,+\infty)$. But for the case 
$L(\alpha)=L(\beta)=(-\infty,+\infty)$, Theorem 2.1 reduces the problem of $G$-equivalence of substantional curves to the $(G, R)$-equivalence of paths.

3. The generating system of the differential field of invariant differential rational functions of a path and the problem of equivalence of curves

Let $x(t)$ be an $I$-path in $R^{n}$.

Definition 3.1. A polynomial $p\left(x, x^{\prime}, \ldots, x^{(k)}\right)$ of $x$ and a finite number of derivatives $x^{\prime}, \ldots, x^{(k)}$ of $x$ with coefficients from $R$ will be called a differential polynomial of $x$. It will be denoted by $p\{x\}[9]$.

We denote the set of all differential polynomials of $x$ by $R\{x\}$. It is a differential $R$-algebra. It is also an integral domain. Therefore there exists a quotient field $R\langle x\rangle$ of $R\{x\}$ and every element of $R\langle x\rangle$ is of the form $f\langle x\rangle=$ $\frac{p\{x\}}{q\{x\}}$, where $p\{x\}$ and $q\{x\} \neq 0$ are differential polynomials of $x$. Any element of $R\langle x\rangle$ will be called a differential rational function of $x$. The derivative operator of $R\{x\}$ can be extended uniquely to $R\langle x\rangle$ as $f^{\prime}\langle x\rangle=\left(\frac{p\{x\}}{q\{x\}}\right)^{\prime}=$ $\frac{p^{\prime}\{x\} q\{x\}-p\{x\} q^{\prime}\{x\}}{(q\{x\})^{2}}$. Let $G$ be a subgroup of $G L(n, R)$.

Definition 3.2. $\quad$ A differential rational function $f\langle x\rangle$ will be called $G$ invariant if $f\langle g x\rangle=f\langle x\rangle$ for all $g \in G$.

The set of all $G$-invariant differential rational functions of $x$ will be denoted by $R\langle x\rangle^{G}$. It is a differential subfield of $R\langle x\rangle$.

Definition 3.3. $\quad$ A subset $S$ of $R\langle x\rangle^{G}$ will be called a generating system of $R\langle x\rangle^{G}$ if the smallest differential subfield containing $S$ is $R\langle x\rangle^{G}$.

Theorem 3.1. The system

$$
\frac{\left[x^{(n)} x^{\prime} \ldots x^{(n-1)}\right]}{\left[x x^{\prime} \ldots x^{(n-1)}\right]}, \frac{\left[x x^{\prime} \ldots x^{(i-1)} x^{(n)} x^{(i+1)} \ldots x^{(n-1)}\right]}{\left[x x^{\prime} \ldots x^{(n-1)}\right]}, i=1, \ldots, n-1,
$$

is a generating system of $R\langle x\rangle^{G}$.

Proof. For the proof, see ([8, p. 79])

Theorem 3.2. $\quad$ Let $\alpha, \beta$ be substantial curves in $R^{n}$ and $x \in \phi_{\alpha}, y \in$ $\phi_{\beta}$. Then,

(i) for $L(\alpha)=L(\beta) \neq(-\infty,+\infty), \alpha \stackrel{G}{\sim} \beta$ if and only if

$$
\begin{aligned}
& \operatorname{sgn} \frac{\left[x^{(n)}(s) x^{\prime}(s) \ldots x^{(n-1)}(s)\right]}{\left[x(s) x^{\prime}(s) \ldots x^{(n-1)}(s)\right]}=\operatorname{sgn} \frac{\left[1 y^{(n)}(s) y^{\prime}(s) \ldots y^{(n-1)}(s)\right]}{\left[y(s) y^{\prime}(s) \ldots y^{(n-1)}(s)\right]} \\
& \frac{\left[x(s) x^{\prime}(s) \ldots x^{(i-1)}(s) x^{(n)}(s) x^{(i+1)}(s) \ldots x^{(n-1)}(s)\right]}{\left[x(s) x^{\prime}(s) \ldots x^{(n-1)}(s)\right]} \\
& =\frac{\left[y(s) y^{\prime}(s) \ldots y^{(i-1)}(s) y^{(n)}(s) y^{(i+1)}(s) \ldots y^{(n-1)}(s)\right]}{\left[y(s) y^{\prime}(s) \ldots y^{(n-1)}(s)\right]}
\end{aligned}
$$


for all $s \in L(\alpha)=L(\beta)$ and $i=1, \ldots, n-1$.

(ii) for $L(\alpha)=L(\beta)=(-\infty,+\infty), \alpha \stackrel{G}{\sim} \beta$ if and only if there exists $b \in(-\infty,+\infty)$ such that

$$
\begin{aligned}
& \operatorname{sgn} \frac{\left[x^{(n)}(s) x^{\prime}(s) \ldots x^{(n-1)}(s)\right]}{\left[x(s) x^{\prime}(s) \ldots x^{(n-1)}(s)\right]}=\operatorname{sgn} \frac{\left[y^{(n)}(s+b) y^{\prime}(s+b) \ldots y^{(n-1)}(s+b)\right]}{\left[y(s+b) y^{\prime}(s+b) \ldots y^{(n-1)}(s+b)\right]}, \\
& \frac{\left[x(s) x^{\prime}(s) \ldots x^{(i-1)}(s) x^{(n)}(s) x^{(i+1)}(s) \ldots x^{(n-1)}(s)\right]}{\left[x(s) x^{\prime}(s) \ldots x^{(n-1)}(s)\right]} \\
& =\frac{\left[y(s+b) y^{\prime}(s+b) \ldots y^{(i-1)}(s+b) y^{(n)}(s+b) y^{(i+1)}(s+b) \ldots y^{(n-1)}(s+b)\right]}{\left[y(s+b) y^{\prime}(s+b) \ldots y^{(n-1)}(s+b)\right]}
\end{aligned}
$$

for all $s \in(-\infty,+\infty)$ and $i=1, \ldots, n-1$.

Proof. (i) Let $\alpha \stackrel{G}{\sim} \beta$. By claim $(i)$ of Theorem 2.1, $x \stackrel{G}{\sim} y$. By Proposition $2.3,\left|\frac{\left[x^{(n)} x^{\prime} \ldots x^{(n-1)}\right]}{\left[x x^{\prime} \ldots x^{(n-1)}\right]}\right|=\left|\frac{\left[y^{(n)} y^{\prime} \ldots y^{(n-1)}\right]}{\left[y y^{\prime} \ldots y^{(n-1)}\right]}\right|=1$. This, in view of $x \stackrel{G}{\sim} y$, yields the formulae (3.1). Now suppose that (3.1) holds. By Proposition 2.3, we have $\left|\frac{\left[x^{(n)} x^{\prime} \ldots x^{(n-1)}\right]}{\left[x x^{\prime} \ldots x^{(n-1)}\right]}\right|=\left|\frac{\left[y^{(n)} y^{\prime} \ldots y^{(n-1)}\right]}{\left[y y^{\prime} \ldots y^{(n-1)}\right]}\right|=1$. Using (3.1), we obtain

$$
\begin{gathered}
\frac{\left[x^{(n)}(s) x^{\prime}(s) \ldots x^{(n-1)}(s)\right]}{\left[x(s) x^{\prime}(s) \ldots x^{(n-1)}(s)\right]}=\frac{\left[y^{(n)}(s) y^{\prime}(s) \ldots y^{(n-1)}(s)\right]}{\left[y(s) y^{\prime}(s) \ldots y^{(n-1)}(s)\right]}, \\
\frac{\left[x(s) x^{\prime}(s) \ldots x^{(i-1)}(s) x^{(n)}(s) x^{(i+1)}(s) \ldots x^{(n-1)}(s)\right]}{\left[x(s) x^{\prime}(s) \ldots x^{(n-1)}(s)\right]} \\
=\frac{\left[y(s) y^{\prime}(s) \ldots y^{(i-1)}(s) y^{(n)}(s) y^{(i+1)}(s) \ldots y^{(n-1)}(s)\right]}{\left[y(s) y^{\prime}(s) \ldots y^{(n-1)}(s)\right]} .
\end{gathered}
$$

Let us consider the matrix

$$
A_{x}(t)=\left\|x(t) x^{\prime}(t) \ldots x^{(n-1)}(t)\right\| .
$$

By the substantiality of $x \operatorname{det} A_{x}(t)=\left[x(t) x^{\prime}(t) \ldots x^{(n-1)}(t)\right] \neq 0$ for all $t$ in $I$. Therefore there exists the matrix $A_{x}^{-1}(t)$. We consider the matrices

$$
A_{x}^{\prime}(t)=\left\|x^{\prime}(t) x^{(2)}(t) \ldots x^{(n)}(t)\right\| \text { and } A_{x}^{-1}(t) A_{x}^{\prime}(t)=\left\|c_{i j}(t)\right\| .
$$

It is easily obtained that

1. $c_{j+1 j}(t)=1$ for all $t$ in $I, j: 1 \leq j \leq n-1$;

2. $c_{i j}(t)=0$ for all $t$ in $I, j \neq n, i \neq j+1,1 \leq i \leq n$;

3. $c_{i+1 n}(t)=\frac{\left[x(t) x^{\prime}(t) \ldots x^{(i-1)}(t) x^{(n)}(t) x^{(i+1)}(t) \ldots x^{(n-1)}(t)\right]}{\left[x(t) x^{\prime}(t) \ldots x^{(n-1)}(t)\right]}$ for all $t$ in $I, 0 \leq$ $i \leq n-1$.

Similarly, for $A_{y}^{-1}(t) A_{y}^{\prime}(t)=\left\|d_{i j}(t)\right\|$ we have

1. $d_{j+1 j}(t)=1$ for all $t$ in $I, j: 1 \leq j \leq n-1$; 
2. $d_{i j}(t)=0$ for all $t$ in $I, j \neq n, i \neq j+1,1 \leq i \leq n$;

3. $d_{i+1 n}(t)=\frac{\left[y(t) y^{\prime}(t) \ldots y^{(i-1)}(t) y^{(n)}(t) y^{(i+1)}(t) \ldots y^{(n-1)}(t)\right]}{\left[y(t) y^{\prime}(t) \ldots y^{(n-1)}(t)\right]}$ for all $t$ in $I, 0 \leq$ $i \leq n-1$.

We obtain from $(3.1)$ that $c_{i j}(t)=d_{i j}(t)$ for all $t$ in $I, i, j=1, \ldots, n$. Then

$$
A_{x}^{-1}(t) A_{x}^{\prime}(t)=A_{y}^{-1}(t) A_{y}^{\prime}(t) .
$$

We have

$$
\begin{aligned}
\left(A_{y} A_{x}^{-1}\right)^{\prime} & =A_{y}^{\prime} A_{x}^{-1}+A_{y}\left(A_{x}^{-1}\right)^{\prime}=A_{y}^{\prime} A_{x}^{-1}+A_{y}\left(-A_{x}^{-1} A_{x}^{\prime} A_{x}^{-1}\right) \\
& =A_{y}\left(A_{y}^{-1} A_{y}^{\prime}-A_{x}^{-1} A_{x}^{\prime}\right) A_{x}^{-1}=0 .
\end{aligned}
$$

Therefore $A_{y}(t) A_{x}^{-1}(t)$ does not depend on $t$. Put $g=A_{y}(t) A_{x}^{-1}(t)$. As $\operatorname{det} A_{x}(t) \neq 0$ for all $t$ in $I$, and $A_{y}(t) \neq 0$ for all $t$ in $I$, then $\operatorname{det} g \neq 0$. We have $A_{y}(t)=g A_{x}(t)$. Therefore $y(t)=g x(t)$ for all $t$ in $I$. Thus $x \stackrel{G}{\sim} y$. The proof of (ii) follows similarly from claim (ii) of Theorem 2.1.

Let $T$ be one of the sets $(0, l)$ (where $l \leq+\infty),(-\infty, 0),(-\infty,+\infty)$.

Theorem 3.3. Let $h_{1}(s), \ldots, h_{n}(s)$ be $C^{\infty}$-functions on $T$, where $\left|h_{1}(s)\right|=1$ for all $s \in T$. Then there exists an invariant parametrization $x$ of a substantial curve such that

$$
\begin{aligned}
& \frac{\left[x^{(n)}(s) x(s) x^{\prime}(s) \ldots x^{(n-1)}(s)\right]}{\left[x(s) x^{\prime}(s) \ldots x^{(n-1)}(s)\right]}=h_{1}(s) \\
& \frac{\left[x(s) x^{\prime}(s) \ldots x^{(i-1)}(s) x^{(n)}(s) x^{(i+1)}(s) \ldots x^{(n-1)}(s)\right]}{\left[x(s) x^{\prime}(s) \ldots x^{(n-1)}(s)\right]}=h_{i+1}(s)
\end{aligned}
$$

for all $s \in T$ and $i=1, \ldots, n-1$.

Proof. Let $C(s)$ be the matrix $\left\|c_{i j}(s)\right\|$, where $c_{j+1 j}(s)=1$ for all $s \in T$, $1 \leq j \leq n-1 ; c_{i j}(s)=0$ for all $s \in T, j \neq n, i \neq j+1,1 \leq i \leq n ; c_{i n}(s)=h_{i}(s)$, $i=1, \ldots, n$. It is known from the theory of differential equations that there exists a solution of the differential equation

$$
A_{x}^{\prime}(s)=A_{x}(s) C(s)
$$

such that $\operatorname{det} A_{x}(s) \neq 0$ for all $s \in T$, where $A_{x}(s)=\left\|x(s) x^{\prime}(s) \ldots x^{(n-1)}(s)\right\|$ is the matrix of column vectors $x(s), x^{\prime}(s), \ldots, x^{(n-1)}(s)$ and $A_{x}^{\prime}(s)=$ $\left\|x^{\prime}(s) x^{\prime \prime}(s) \ldots x^{(n)}(s)\right\|$ is the matrix of column vectors $x^{\prime}(s), x^{\prime \prime}(s), \ldots, x^{(n)}(s)$. Let $A_{x}(s)$ be such solution of the differential equation (3.3). From (3.3) we have $A_{x}^{-1}(s) A_{x}^{\prime}(s)=C(s)$. From this equation we get the equalities (3.2)

Remark 3. The functions

$$
\frac{\left[x(s) x^{\prime}(s) \ldots x^{(i-1)}(s) x^{(n+1)}(s) x^{(i+1)}(s) \ldots x^{(n)}(s)\right]}{\left[x(s) x^{\prime}(s) \ldots x^{(n)}(s)\right]},
$$

where $i=1, \ldots, n$, equal to the functions $c_{1}(s), \ldots, c_{n}(s)$ of Gardner and Wilkens $([5$, p. 398]). 


\author{
DePARTMENT OF MATHEMATiCS \\ Karadeniz TEChNiCAL UnIVERSITY \\ 61080, Trabzon, TURKEY \\ e-mail: peksen@ktu.edu.tr \\ djavvat@yahoo.com
}

\title{
References
}

[1] E. De Angelis, T. Moons, L. Van Gool and P. Verstraelen, Complete systems of affine semi-differential invariants for plane and space curves, In: Dillen, F.(ed.) et al., Geometry and topology of submanifolds, VIII, Proceedings of the international meeting on geometry of submanifolds, Brussels, Belgium, July 13-14, 1995 and Nordfjordeid, Norway, July 18August 7, 1995. Singapore: World Scientific, 1996, 85-94.

[2] W. Barthel, Zur affinen Differentialgeometrie -Kurventheorie in der allgemeinen Affingeometrie, Proceedings of the Congress of Geometry, Thessaloniki (1987), 5-19.

[3] W. Blaschke, Affine Differentialgeometrie, Berlin, 1923.

[4] E். Cartan, La théorie des groupes finis et continus et la géométrie différentielle, Gauthier-Villars, Paris, 1951.

[5] R. B. Gardner and G. R. Wilkens, The fundamental theorems of curves and hypersurfaces in centro-affine geometry, Bull. Belg. Math. Soc. 4 (1997), 379-401.

[6] H. W. Guggenheimer, Differential Geometry, McGraw-Hill, New York, 1963.

[7] S. Izumiya and T. Sano, Generic affine differential geometry of space curves, Proceedings of the Royal Society of Edinburg 128A (1998), 301314 .

[8] D. Khadjiev, The Application of Invariant Theory to Differential Geometry of Curves, Fan Publ., Tashkent, 1988.

[9] D. Khadjiev and Ö. Pekşen, The complete system of global differential and integral invariants for equi-affine curves, Differential Geometry and its Applications 20 (2004), 167-175.

[10] W. Klingenberg, A Course in Differential Geometry, Springer-Verlag, New York, 1978.

[11] D. Laugwitz, Differentialgeometrie in Vectorraumen, Friedr. Vieweg \& Sohn, Braunschweig, 1965.

[12] K. Nomizu and T. Sasaki, Affine Differential Geometry, Cambridge Univ. Press, 1994. 
[13] H. P. Paukowitsch, Begleitfiguren und Invariantensystem minimaler Differentiationsordnung von Kurven im reellen $n$-dimensionalen affinen Raum, Mh. Math. 85-2 (1978), 137-148.

[14] E. Salkowski, Affine Differentialgeometrie, W. de Gruyter, Berlin, 1934.

[15] P. A. Schirokow and A. P. Schirokow, Affine Differentialgeometrie, Teubner, Leipzig, 1962.

[16] U. Simon and W. Burau, Blaschkes Beitrage zur affinen Differentialgeometrie, In: W. Blaschke (ed.), Gesammelte Werke IV (1985), 11-34.

[17] U. Simon, Entwicklung der affinen Differentialgeometrie nach Blaschkes, In: W. Blaschke (ed.), Gesammelte Werke IV (1985), 35-88.

[18] _ Recent developments in affine differential geometry, Diff. Geom. and its Applications, Proc. Conf. Dubrovnik/Yugosl. 1988, 1989, 327-347.

[19] U. Simon, H. L. Liu, M. Magid and Ch. Scharlach, Recent developments in affine differential geometry, In: Geometry and Topology of Submanifolds VIII, World Scientific, Singapore, 1966, 1-15 and 293-408.

[20] B. Su, Affine Differential Geometry, Science Press, Beijing, Gordon and Breach, New York, 1983.

[21] A. M. Suhtaeva, On the equivalence of curves in $C^{n}$ with respect to the action of groups $S L(n, C)$ and $G L(n, C)$, Dokl. Akad. Nauk of SSRUz, N6 (1987), 11-13. 